Kai A. Konrad

\title{
Search Duplication in Research and Design Spaces - Exploring the Role of Local Competition
}

\author{
Max Planck Institute for Tax Law and Public Finance \\ Working Paper 2014 - 19
}

October 2014

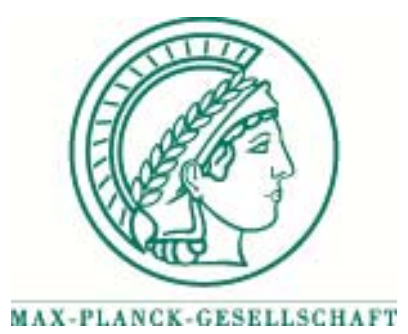

Max Planck Institute for

Tax Law and Public Finance

Department of Business and Tax Law

Department of Public Economics

http://www.tax.mpg.de 
Working papers of the Max Planck Institute for Tax Law and Public Finance Research Paper Series serve to disseminate the research results of work in progress prior to publication to encourage the exchange of ideas and academic debate. Inclusion of a paper in the Research Paper Series does not constitute publication and should not limit publication in any other venue. The preprints published by the Max Planck Institute for Tax Law and Public Finance represent the views of the respective author(s) and not of the Institute as a whole. Copyright remains with the author(s).

Max Planck Institute for Tax Law and Public Finance

Marstallplatz 1

D-80539 Munich

Tel: $\quad+498924246-0$

Fax: $\quad+498924246-501$

E-mail:ssrn@tax.mpg.de

http://www.tax.mpg.de 


\title{
Search duplication in research and design spaces - exploring the role of local competition
}

\author{
Kai A. Konrad* \\ Max Planck Institute for Tax Law and Public Finance \\ and Department of Economics, University of Munich
}

February 17, 2014

\begin{abstract}
Clustering and lack of sufficient diversification in research strategies has been identified as an important problem for delegated research as it takes place in design contests by Erat and Krishnan (2012). We show that this problem can be solved by local competition (such as bribery, lobbying or rent seeking) among players who apply the same search strategies or develop the same design. Such competition can restore full efficiency in the non-cooperative equilibrium. Local competition interacts with the choice of whether to cluster or to diversify, and rather than adding a further inefficiency to the existing ones, it eliminates inefficiency. The results are robust and hold under simultaneous search strategy choices as well as for sequential choices.
\end{abstract}

JEL classification code: O32, O33, D72, D74

Keywords: Delegated research, clustering, product design, design contest, search strategy, rent-seeking

\footnotetext{
*Correspondence address: Kai A. Konrad, Max Planck Institute for Tax Law and Public Finance, Marstallplatz 1, 80539 Munich, Germany. E-mail: kai.konrad@tax.mpg.de. I thank Florian Morath for helpful comments. The usual caveat applies.
} 


\section{Introduction}

Diversification in delegated or decentralized research and the problem of possible clustering is an important dimension in contests about ideas and design: researchers need to decide where they search in the space of possible designs. They should search at different sites to reduce duplication of effort. ${ }^{1}$ Erat and Krishnan (2012) [E\&K in what follows] analyze this problem. They find that clustering in the same 'search site' may occur naturally in the equilibrium and that too little diversification may occur. ${ }^{2}$ They suggest multiple prizes to partially improve the effectiveness of delegated research. ${ }^{3}$ We take up their framework and stay close to it, but allow for competition that emerges if several searchers arrive at the same 'site' leading to the same idea or design. The possibility of this local competition drastically changes the nature of the equilibrium and yields a first-best efficient allocation of search: it eliminates clustering, avoids duplication of search strategies and leads to efficient diversification. In addition, it yields efficient search effort choices. The results hold for equilibrium with simultaneous choices as well as for sequential choices. Hence, this paper shows that delegated design research may perform well: The choice of search sites and competition that emerges inside the same site are complementary activities. While each of them is a possible source of inefficiency, their interaction leads to an efficient outcome.

The problem of parallel research has been explored in a number of contexts. Early on, duplication of effort has been emphasized in the related field of patent races (see, e.g., Loury (1979) and Lee and Wilde (1980)). If several researchers follow the same approach and choose positive effort to preempt the competitors in a patent race, then this may lead to a duplication of effort. The total sum of efforts expended by each contestant may also be too large. The survey by Adamczyk et al. (2012) documents the large and increasing

\footnotetext{
${ }^{1}$ Chatterjee and Evans (2004) highlight prominent examples. Sony and JVC both expended effort on different technologies for recording systems for marketable video players, and only one system was awarded the economic rent attached to this market.

${ }^{2}$ The issue of diversification in research strategies is mentioned or touched upon in work by Rosen (1986), Hvide (2002), Hvide and Kristiansen (2003), Gaba, Tsetlin and Winkler (2004), who focus on the choice of stochastic success-interdependence of the contestants' efforts. Related questions are addressed by Loch, Terwiesch and Thomke (2001), who consider parallel versus sequential testing of design alternatives.

${ }^{3}$ Multiple prizes have traditionally been analyzed in the context of choosing how much research effort to expend. Pioneering work is by Glazer and Hassin (1988), Clark and Riis (1996, 1998), Moldovanu and Sela (2001, 2006), Szymanski and Valletti (2005). Unlike Erat and Krishnan (2012), these approaches focus on 'how much' to search. They disregard the question of 'where' to search.
} 
importance of delegated search for ideas or design solutions in a more structured process that gives a specified set of prizes to the winner of a tournament, typically described as innovation contests, and Hutter et al. (2011) illustrate the role of different forms of interaction between the contestants in such set-ups. The issue of where to search - rather than the issue of how much to search - and the role of locational choice in the space of search approaches as a strategic variable is more explicitly studied in a few, more abstract papers. Fershtman and Rubinstein (1997) look at a situation in which there are several sites. A prize is hidden in one of these sites. They consider two competitors, each of whom can choose several sites and chooses how many sites to visit, and then choose the sequencing of search, assuming that they scrutinize one site after another. Chatterjee and Evan (2004) also focus on two competitors, but they analyse a different dynamic structure. Cardon and Sasaki (1998) analyze a similar problem in the context of patent races. They focus on how competitors sequence the visits of a number of sites and find that the strategic interaction between the contestants may induce them to cluster in how they sequence the search process. They study a structure by which the development of patents that are imperfect substitutes are feasible, and discuss how downstream competition affects the sequencing choice. They also touch upon the relationship between clustering and downstream competition, using a framework with two possible levels of $R \& D$ research intensity. Their policy focus is on the desirability of $R \& D$ subsidies in patent races. The framework that is closest to ours is E\&K (2012). They consider multiple contestants in delegated research in design contests with many possible search sites of heterogeneous quality. Quality of a site in their context is defined by the likelihood for the design alternative that is found at this site to be the one that is most preferred by the designer of the contest. In the context of design contests, it is consistent to assume that each competitor can choose one site only (i.e., turn in only one proposal). They find an inefficient choice of sites with search duplication in some sites and lack of search in other sites, even if the participants can coordinate.

The results by E\&K (2012) are the starting point of our analysis. We allow for a further type of interaction: competition among players who choose the same site. We show that the competition that may emerge naturally between those contestants who cluster at the same site can completely solve the duplication problem in decentralized design research and leads to a first-best efficient outcome. Our approach is not specific regarding the source of this competition. Competition may emerge along the dimension of small quality improvements that allow the contest procurer to make a choice if several players offer the same design or 
idea. Competitors at the same 'site' may also lobby the procurer: they may engage in bribery or in a rent-seeking contest and expend efforts to influence the procurer's decision. ${ }^{4}$ Also, players arriving at the same site may start quarreling or fighting about who owns the site. Such issues have received considerable attention in the theory of conflict (see, e.g., Skaperdas 1982, Garfinkel and Skaperdas 2012). Finally, the competition between players offering the same idea or design may be staged more formally by the procurer. The procurer may adopt an all-pay auction or another competitive process to allocate the prize between players who chose the same design/site. The adoption of such an incentive instrument is another means by which the procurer influences the choice of 'where to search' and by which the problem of clustering in delegated design contests can be solved.

\section{A formal analysis}

We consider a 'space' $M$ consisting of a finite number $m$ of different design 'search sites' $j$. Depending on the specific application, sites could be seen as a disjunct set of 'designs' or 'ideas', like in an architecture competition, as discussed in E\&K (2012), with ex-ante uncertainty about which design appears most attractive in the view of the procurer. ${ }^{5}$ We use a metaphorical geographical interpretation: $M$ is a partition of the design landscape into sites $j=1,2, \ldots, m$, where searchers have to decide which site they choose for their search. Each searcher can choose only one site. The procurer of the design contest awards one well-specified prize. More formally, the award winning 'idea' is located in at most one

\footnotetext{
${ }^{4}$ Suppose searchers generate the same idea or design, but only one of them can be given the prize. They may expend effort ('wining and dining', competition for attention, bribes) to influence the decision-maker in their favor. Such effort may be ineffective as regards a choice between substantially different designs, but may be particularly relevant for designs that are almost indistinguishable from an objective point of view. This type of rent-seeking has been explored extensively in many contexts. See Tullock (1967, 1980) for early contributions, Konrad (2009) for an extensive survey, and Congleton et al. (2008) for a two-volume reprint collection of most important papers.

${ }^{5}$ Research duplication is welfare-reducing in our (E\&K's) framework. For an empirical account of the role of the number of competitors for the trade-off between encouraging innovation speed and avoiding duplication effort, see Boudreau, Lacetera and Lakhani (2011). Simmonds (1985) discussed whether research duplication is good or bad. He uses the context of U.K. potato breeding to suggest that research approaches by the Scottish Crops Research Institute and by the Plant Breeding Institute, Cambridge, may have started with similar objectives, but their research led to innovations that are both useful and complementary to each other. His example hints at the fact that 'programs with similar titles and objectives are likely to take different routes and to attack problems in different styles ...' (Simmonds, 1985, 55).
} 
of the non-overlapping sites. All other sites are, in that sense, empty and searching there is futile. The prize itself has an economic value and this prize value is normalized and equal to 1 and is the same for all searchers. The ex-ante probabilities for the different sites to be the prize-carrying site are

$$
p_{1} \geq p_{2} \geq \ldots \geq p_{m}, \text { with } p=\Sigma p_{j} \leq 1
$$

This describes that at most one site hides a prize, but we do not rule out the case that all sites are empty, which happens with a probability of $(1-p)$.

Consider now searchers, their possible actions and outcomes. The set of agents who may become active as searchers is $N=\{1,2, \ldots, n\}$. If an agent decides to stay out, the agent receives a payoff of zero. This is a normalization. If an agent decides to enter, he or she must choose one and only one search site. Choice of a given site $j$ causes a fixed cost $c$, which is the same for all sites. First, we consider simultaneous choices and non-cooperative coordination equilibrium, following E\&K (2012). Perfect coordination with simultaneous decisions is a strong assumption. In a later section, we discuss why this assumption is not crucial: the results receive strong support from other timing structures.

Once all players decided whether to become active and which site to choose, each active searcher $i$ must also choose competition effort $x_{i j} \geq 0$. As discussed in the introduction, this effort may be seen as rent-seeking (influence activities, bribery, lobbying) effort of searchers locating in the same site, but it may also have a number of other interpretations. Jointly with the site cost $c$, this yields a total expenditure of $c+x_{i j}$. Once these decisions are made, the searchers learn which site wins the prize. If a player searches in a site that does not carry the prize, then the expenditure $c+x_{i j}$ is lost. The search effort $x_{i j}$ at site $j$ determines $i$ 's competitive edge compared to other players searching at the same site. If there is only one searcher $i$ at site $j$, then $i$ will eventually find the prize if the prize is there, and appropriate its full benefit of 1 . If there are several players searching at the prize-carrying site $j$, then the one who expends the highest effort $x_{i j}$ receives the prize. If several players at this site expend exactly the same highest effort, then they all win with equal probability. All non-winning searchers at the same or other sites pay their cost but receive zero reward.

The choice of rent-seeking effort that can improve a player's competitive situation at the same site is the main departure from E\&K (2012). This departure turns out to be crucial. E\&K (2012) find considerable potential for inefficiency. Inefficiency is due to a wrong number of entries and misallocation of the active set of searchers among the existing sites. One might expect that an option to expend additional effort $x_{i j}$ that improves a searcher's own position 
in a given location may cause further inefficiency, particularly if $x_{i j}$ is wasteful from a welfare point of view. However, as we show, the option to expend such wasteful competition effort restores full efficiency. It eliminates both the problem of duplication and the problem of not searching in sites that are still sufficiently valuable from an efficiency point of view.

We made some important assumptions. First, we assumed that the size of the prize is given. This assumes that the duplication effort is simply wasteful - which is in line with the general framework by E\&K (2012). Second, we assume that the winner among the players in the prize-carrying site is determined in an all-pay auction without noise: the player in the non-empty site who expends the highest effort wins the prize. Our main results also hold for a large set of alternative allocation rules that determine who among the searchers locating in the same site wins the prize. These other mechanisms include other types of tournament competition with noise such as Tullock's (1980) lottery contest and tournaments with additive noise as in Lazear and Rosen (1981). For the result to hold it is required that the equilibrium amount of effort that is expended by players in the same site is sufficiently high. Third, we assume common knowledge. In particular, it is important that searchers know whether they have competitors searching on the same 'design site'. This is an important assumption. Knowledge about possible competitors is vital for the efficiency result. The vital role of information about competitors hints at important policy implications.

First-best efficiency Social welfare among contestants in this framework is described by the sum of rents, and for a given number of entrants $n_{j}$ in each different site $j \in M=\{1, \ldots m\}$ and players' efforts $x_{i j}$, this sum can be expressed as

$$
Q=\sum_{j=1}^{m} I(j) p_{j}-\sum_{j=1}^{m} n_{j} c-\sum_{j=1}^{m} \sum_{i=1}^{n} x_{i j} .
$$

Here $I(j)$ is an indicator variable taking the value of 1 if $n_{j}>0$ and the value 0 if $n_{j}=0$. So the first expression is the social value of the innovation (normalized to 1 in here) times the sum of probabilities of prize worthy discovery for all sites that attract at least one searcher. ${ }^{6}$ The second term is the sum of fixed costs that emerge from searchers searching a site. The third term sums up all players' competition efforts.

\footnotetext{
${ }^{6}$ One may attribute a higher social welfare $\theta>1$ to the innovation. In this case the social benefit would be $\sum_{j=1}^{m} I(j) \theta_{j} p_{j}$. This shows that, unless the size of the prize is also adjusted upwards, there will be search in too few sites.
} 
Proposition 1 The efficiency benchmark is characterized as follows. (i) If $n$ is at least as large as the number of sites $j$ for which $p_{j}>c$, then the maximum of $Q$ is obtained if all sites that have $p_{j}>c$ attract precisely one searcher. (ii) If $n$ is smaller than the number of sites $j$ with $p_{j}>c$, then each of the $n$ sites with the highest $p_{j}$ should attract exactly one searcher, until the number of searchers is exhausted. The optimal amount of effort is $x_{i j}=0$ for all players at all sites in both cases (i) and (ii).

The proposition describes the efficiency benchmark and follows straightforwardly from (2). We can later use this benchmark to compare the different equilibria with respect to their efficiency properties. Three sources of inefficiency can be distinguished. First, multiple players may cluster at the same site, causing duplication costs. Second, some sites with $p_{j}>c$ may attract no searchers, causing insufficient entry or insufficient diversification in the search strategies. Third, players may expend competition effort, which in our set-up is wasteful from a social point of view. We will see that E\&K (2012) give room for the first two types of inefficiency to arise. We will also see that the introduction of the possibility of the third type of inefficiency (in-site competition), rather than inducing further inefficiency, brings the system back to full efficiency.

Entry-cost only We first reconsider the set-up for which $x_{i j} \equiv 0$. This set-up reproduces the equilibrium of E\&K (2012), confirming their results on duplication and insufficient diversification. Their main result can be restated for the specific set-up here:

Proposition 2 Let competition effort be ruled out $\left(x_{i j} \equiv 0\right)$. (i) If $n$ is sufficiently large then the equilibrium search allocation in pure strategies is characterized by $n_{j}=\operatorname{int}\left(p_{j} / c\right)$ searchers choosing site $j$ for all $j=1, \ldots m$. Here, $n_{j}=\operatorname{int}\left(p_{j} / c\right)$ is the largest integer smaller than $p_{j} / c$. (ii) If $n$ is smaller than $\Sigma_{j} n_{j}$ as defined in (i), then all $n$ players enter and allocate in sites that form a subset $Z \subseteq M$. For each two sites $j \in Z$ and $g \in Z$ from this subset it holds that

$$
\frac{p_{j}}{n_{j}} \geq \frac{p_{g}}{n_{g}+1}
$$

For all sites $h \in M \backslash Z$ that attract no player it holds that,

$$
\min \left\{\frac{p_{j}}{n_{j}} \mid j \in Z\right\} \geq p_{h}
$$

A formal proof could basically follow E\&K (2012) and is not repeated here. 
Intuitively, if more players enter into one site, they drive down the expected profit per player that is earned by the players entering into this site. If there is no shortage of possible entrants, then each site attracts entrants exactly up to the point at which further entry is no more profitable. ${ }^{7}$ Sites at which the first entry is already not profitable remain empty. This describes in words what is expressed more formally in the proposition in (i). For all sites with $p_{j}>2 c$, for instance, free entry implies research duplication. Each of these sites attracts more than one player. For some sites with $p_{j} \in(c, 2 c]$ the equilibrium condition in (i) means that exactly one searcher enters into this site. For sites with very low $p_{j} \leq c$, this means that entry into this site is not profitable, not even for the first searcher who may consider entering there and who would be the only searcher in that site.

If the solution just characterized requires more searchers than the $n$ potential searchers that exist, then the number of players becomes a binding constraint. In this case the players will earn a scarcity rent. The size of this rent may differ among the different sites, due to the integer problem, but the rent will be such that no searcher can increase his own payoff by leaving his current site and entering into another site. This is what condition (3) describes. Also, the marginal site will typically be characterized by a $p_{j}$ higher than $c$, and this is described by (4). The outcome in (ii) implies that, even though there may be duplication in some sites, other sites that are profitable would remain empty, which refers to the second type of inefficiency described in the welfare section.

Competition effort Now we turn to the case with endogenous efforts $x_{i j} \geq 0$, by which a searcher $i$ who has chosen to locate at site $j$ can outcompete other players who enter the same site. The game has the following timing. In stage 1 each potential searcher $i \in N$ chooses whether to stay out or to enter, and in the latter case, which site to enter. A potential searcher who stays out receives a payoff of zero and also chooses zero effort. A searcher who chooses a site and enters, expends an entry fee $c$. These choices are irreversible and the entry fee is sunk at this stage and is not refundable. Now all searchers find out how many other players arrived at the same site. At stage 2 all searchers $i$ who entered a given site $j$ simultaneously choose $x_{i j} \geq 0$. Allowing for these costs of outcompeting others, we first consider the non-cooperative coordination equilibria in pure strategies that correspond to the

\footnotetext{
${ }^{7}$ Generically, the inequality is fulfilled with strict inequality. For the non-generic case of equality we make a tie-breaking assumption: a searcher stays out if he is just indifferent as to whether to enter or not. This tie-breaking assumption is chosen because it increases the efficiency of the equilibrium outcome whenever it becomes relevant.
} 
equilibria described in Proposition 2.

Proposition 3 If non-negative effort to compete with others at the same site can be chosen endogenously, then the non-cooperative coordination equilibria in pure strategies are all characterized by a first-best allocation of players to sites and efficient choices of competition effort.

Proof. More formally, we have to consider two cases. (i) For unconstrained $n$, i.e., for $n$ larger than the number of sites $j$ for which $p_{j}>c$, we have to show that efficient entry occurs such that there is exactly one entrant per site for all sites with $p_{j}>c$ and zero players enter sites for which $p_{j} \leq c$. (ii) For $n$ smaller than the number of sites $j$ for which $p_{j}>c$, we have to show that players locate in the subset of $M$ which consists of those sites that have the largest difference $p_{j}-c$, with one single player entering each of these sites. Moreover, we have to show that zero competition effort is expended in these equilibria: $x_{i j}=0$ for all $i \in N$ and $j \in M$.

Consider first stage 2. If there is only one entrant at site $j$, then this entrant $i$ maximizes his or her own payoff by $x_{i j}=0$. This player $i$ 's expected payoff is $p_{j}-c$. If the number of entrants at site $j$ is an integer $n_{j}$ with $n_{j}>1$, then these entrants compete for the expected prize at site $j$, using competition efforts $x_{i j}$. By design, the player who expends the highest effort wins the prize if the site carries the prize. The prize is normalized to a value of 1 and the same for all players and the probability for the site to carry the prize is $p_{j}$. All this is common knowledge. Accordingly, stage 2 describes an all-pay auction among $n_{j}$ players for a prize of size $p_{j}$. All players can make non-negative competition efforts $x_{i j}$ and have symmetric costs. This subgame was solved by Baye, Kovenock and deVries (1996). Their Theorem 1 states that for $n_{j}=2$ the equilibrium is unique. For $n_{j}>2$ there is a multiplicity of equilibria, but all equilibria are payoff-equivalent. All equilibria have in common that the expected payoff of each of the $n_{j}$ players at the same site is zero (see Baye et al., 1996, p. 293). ${ }^{8}$ At stage 1 players can anticipate that their payoff in stage 2 is zero if they end up in

\footnotetext{
${ }^{8}$ The result can be made intuitive for the case $n_{j}=2$. First, players would not expend more than $p_{j}$ whichis the expected value of the prize. Second, for any positive choice $x_{1 j}<p_{j}$, player 2 would overbid player 1 , making this positive choice suboptimal for player 1 . But $x_{1 j}=0$ and $x_{1 j}=p_{j}$ can also be shown to be suboptimal for player 1 , given the optimal replies by player 2 to such choices. Hence, the equilibrium must be in mixed strategies. Third, the mixed-strategy equilibrium is characterized by cumulative distribution functions $F_{i}\left(x_{i j}\right)=x_{i j} / p_{j}$ with support [0, $\left.p_{j}\right]$ : If player 1 chooses this mixed strategy, player 2 is indifferent about $x_{2 j} \in\left[0, p_{j}\right]$. To confirm this, each of these choices $x_{2 j}$ yields a stage-2 payoff of $F\left(x_{2 j}\right) p_{j}-x_{2 j}=0$.
} 
the same site with other players in stage 2. In this case, a player's total expected payoff is equal to the entry cost: $0-c=-c<0$.

We now use these expected stage-2 payoffs to solve for the location choices in stage 1 for the case with large $n$. Consider player $i$ who chooses whether to enter and where. The player anticipates the choices by others. Let the choices of all players other than $i$ be such that one searcher plans to enter at each location for which $p_{j}>c$, and no searcher plans to enter a location for which $p_{j} \leq c$. In this case, player $i$ is better-off if he does not enter. Any site at which $i$ expects entry by at least one other player is not attractive. If he enters there, the equilibrium payoff for him will be $-c$. At any site with $p_{j} \leq c$, entry is also not profitable, even if $i$ were the only player who enters there. If, however, there are one or several sites with $p_{j}>c$ and with no other player entering such sites, then it is optimal for $j$ to enter the site with the largest $p_{j}$ among these. Accordingly, unless the number of sites with $p_{j}>c$ exceeds $n$, all sites $j$ with $p_{j}>c$ will be filled with exactly one player, and all other players stay out.

Let us now turn to (ii). Suppose the number of sites with $p_{j}>c$ exceeds $n$. By the same reasoning as before the auction makes entry of multiple players at the same site unprofitable. In stage 1, anticipating if and where other players enter, players are better off if they enter one of the sites with $p_{j}>c$ that otherwise remains empty. And no player will enter a site that has lower $p_{j}-c$ than other, unpopulated sites with higher-probability $p_{j}$. So all players will enter the most profitable sites. This leads to optimal entry, optimal dispersion and zero competition effort.

Intuitively, competition at sites with duplication drives the entry process away from duplication. If several players who enter the same site have to compete with each other, this competition dissipates resources. Formally, we have chosen to describe this competition by the all-pay auction without noise. In this case the prize is fully dissipated in the local competition. This means that players cannot even recover their original investment cost $c$. As a result, the threat of potential competition between multiple entrants at the same site leads to a fully efficient allocation. It avoids duplication as well as lack of diversification. In addition, as competition effort is wasted only if duplication occurs, this effect also avoids wasteful competition efforts.

We have discussed the nature of this competition in the introduction. The competition between searchers at the same site may be stimulated by the desire to be awarded Hence, $F\left(x_{i j}\right)$ are mutually optimal replies to each other. 
the prize in a situation in which the procurer chooses between objectively indistinguishable prize candidates. We highlighted the potential role of rent-seeking and bribing. Also, the procurer may use this situation as an opportunity to maximize his own rent and actively stage a contest between such identical prize candidates. We did not introduce the procurer as an active player. If we do we could give the procurer the option to announce early on, prior to location choices, that players at the same site have to take part in an all-pay contest. If several players choose the same site and if this site carries the prize, an all-pay auction between them determines who wins it. The procurer could implement efficient dispersion by such a choice and still have zero competition efforts in the equilibrium. Competition efforts could be money given to the procurer as a bribe or it could be other, socially more wasteful effort. For the outcome this does not matter as these efforts are zero in the equilibrium.

The result is robust with respect to a number of aspects. First, note that, for the formal analysis, we assumed that competition emerges prior to finding out which site is the prize-carrying site. However, the equilibrium outcome carries over if the intra-site competition takes place only once the searchers at the prize-carrying site have found out that this site is prize-carrying. In this case, only the searchers at this site compete with each other. The prize they compete for is then not $p_{j}$ but equal to 1 , and no competition emerges in all other sites as the prize in these other sites has been found out to be equal to zero. The logic of Baye et al. (1996) implies that the players at the prize-carrying site dissipate the full value of the prize if there is more than one searcher at this site. This dissipation prevents players from clustering at the same site, for the same reason as in Proposition 3. From an ex-ante point of stage 1 , the dissipation that occurs with this structure of timing generates the same expected stage-2 payoff and avoids duplication as in Proposition 3.

Second, the all-pay auction without noise has full dissipation of the value of the prize. Competition between players who cluster at the same site in stage 2 may have less than full dissipation. But it turns out that dissipation need not be complete to reach a fully efficient equilibrium with efficient dispersion in stage 1 . It is sufficient for the efficiency result that the dissipation that emerges in the stage-2 equilibrium with $n_{j} \geq 2$ be high enough to make clustering unattractive. With some notational effort, what is meant by 'sufficient' could be stated more formally. ${ }^{9}$

\footnotetext{
${ }^{9}$ In short, let $a_{j}\left(n_{j}\right) / n_{j}$ be the expected payoff of each player in stage 2 in site $j$ if $n_{j} \geq 1$ players choose this site. Here $a_{j}\left(n_{j}\right)$ is the total expected payoff net of equilibrium competition efforts for all $n_{j}$ players who arrive at $j$. Let $a_{j}\left(n_{j}\right)$ be non-increasing in $n_{j}$. To avoid clustering, the sufficient condition is $a_{j}(2) / 2<c$.
} 
Third, another concern that needs to be raised is the problem of coordination. E\&K (2012) focus on non-cooperative equilibrium with coordination among the potential searchers. This is the right approach given that they show that inefficiency emerges even though potential searchers coordinate their choices. And given that we analyze the implications of intra-site competition for their results, for comparability reasons we make the same assumption here. However, from a more general perspective, simultaneous site choices with perfect coordination may be rather difficult to achieve. As has been discussed in Konrad and Kovenock (2012) in a somewhat different, but structurally related context, the set of pure-strategy equilibrium outcomes with coordination and the set of mixed-location-choice equilibrium outcomes without coordination have different properties. We would like to justify the coordination approach taken by E\&K (2012) and in our enriched framework. We do this by showing that sequential choices on sites lead to the same equilibrium outcomes.

\section{Sequential choices}

In this section, we consider a simple mechanism that generates coordination: players' sequential choice of sites in stage 1. Formally, we replace the simultaneous choice of whether and where to enter in stage 1 by a sequential choice: player 1 decides first whether to enter, and if, which site. Player 2 is next, and so on until player $n$. We need to distinguish between the set-up with $x_{i j} \equiv 0$ and the set-up with endogenous choices of $x_{i j} \geq 0$ that take place once all players made their entry and location decisions. We focus on the case of large $n$.

First we treat the case without intra-site competition effort.

Proposition 4 Let competition effort be ruled out $\left(x_{i j} \equiv 0\right)$. Let players choose sequentially where (and if) to search. The pure-strategy equilibrium that results is an element of the set of non-cooperative coordination equilibria that were characterized in Proposition 2. Let sites be sorted and renumbered according to $\pi_{j} \equiv p_{j} / n_{j}$ in descending order of $\pi_{j}$. The players fill each single site until it is populated by $n_{j}$ players. This process occurs in the sequence of decreasing $\pi_{j}$.

Proof. Given the large number of possible decision paths, a straightforward solution by backward induction is too cumbersome. So we resort to a different argument, making a

For instance, in case of the all-pay auction without noise, $a_{j}(2)=0$, such that this condition always holds, but this is clearly more competition than what is needed to satisfy $a_{j}(2) / 2<c$. 
number of observations that partially build on each other. Recall the definition of $n_{j} \equiv$ $\operatorname{int}\left(p_{j} / c\right)$. (i) No further players will ever enter a site if $n_{j}$ players have already entered this site. This holds because, by definition of $n_{j}$, we have $p_{j} /\left(n_{j}+1\right) \leq c$. This choice is (weakly) inferior to staying out, as the associated payoff is not higher (and generically lower) than the payoff from staying out. (ii) It follows from (i) that each site with positive $n_{j}$ will offer a positive payoff to all players entering this site. (iii) Players will not stay out if there is at least one site $j$, which less than $n_{j}$ players entered. This is true because, in the end, this site will not attract more than $n_{j}$ players by (i) and therefore it offers a positive payoff. This positive payoff makes this choice superior than staying out. (iv) As there are enough potential entrants by assumption, the observations (i), (ii) and (iii) imply that the entry process stops if and only if all sites $j$ are filled with exactly $n_{j}$ players. (v) Players who choose early and consider entering can anticipate the final payoff they reach in each of the $m$ sites: the payoff in site $j$ is equal to $\left(\frac{p_{j}}{n_{j}}-c\right)$ for entrants at this site if $n_{j} \geq 1$. This follows from (iv). And this logic applies for all $j \in M$. When making a choice, a player chooses the site with the highest equilibrium payoff $\left(\frac{p_{j}}{n_{j}}-c\right)$ that is still populated by less than $n_{j}$ players. This, in turn, implies that the sites fill up to their equilibrium populations $n_{j}$ one after the other. Players who choose early anticipate the equilibrium payoffs for each site. Hence, they start with the site that offers the highest equilibrium payoff $\left(\frac{p_{j}}{n_{j}}-c\right)$ for all $j \in M$, followed by the site with the second-highest equilibrium payoff and so on up to the site that offers the smallest positive equilibrium payoff.

Given the complexity of the combinatorial problem and the different decision paths which could be taken by the sequence of players, the proof for the sequential equilibrium is surprisingly simple. It makes use of the properties of the equilibrium payoff players can achieve at each of the sites. Note that players do not necessarily enter the site with the highest $p_{j}$ first. They choose the site that, given the integer problem, offers the largest equilibrium payoff. Note also that the equilibrium that emerges in this sequential game is one of the (many) coordination equilibria that have been characterized by E\&K (2012). This is a strong justification for their focus on coordination equilibria.

Turning now to the case with $x_{i j} \geq 0$, we can again identify the efficiency restoring effect of intra-site competition. Again, we concentrate on the case with $n$ sufficiently large.

Proposition 5 Let intra-site competition effort $x_{i j} \geq 0$ be chosen in stage 2. The sequential non-cooperative equilibria are all characterized by full efficiency: There will be one entrant in 
each site for which $p_{j}>c$, and no entrants for sites with $p_{j} \leq c$. Moreover, zero competition effort is expended: $x_{i j}=0$. If $p_{j}>p_{h}>c$, then site $j$ will attract a searcher prior to site $h$. And if $p_{1}>p_{2}>\ldots .>p_{k}$ for all sites $j=1, \ldots, k$ with $p_{j}>c$, then the sequential equilibrium is unique, with the sites 1 to $k$ being filled in this order.

Proof. The set of possible subgames in stage 2 is exactly the same and they have the same equilibrium payoffs as for simultaneous choice in stage 1. In particular: (i) As shown in the proof of Proposition $3, n_{j} \geq 2$ yields a subgame payoff that is equal to $-c$ for all players in this site. (ii) In turn, this implies that with sequential choice, no player would ever choose a site which is already populated by at least one other player. (iii) This, in turn, implies that the entry stage is completed once a set $Z$ of sites is populated by exactly one player, and a set $M \backslash Z$ of sites remains deserted. (iv) In stage 1 players can base their decision on property (iii). If it is player $i$ 's turn, and if the player finds a positive number of empty sites with $p_{j}-c>0$, the player chooses the one among those sites, which offers the highest $p_{j}-c$. This relies on observation (iii): the player $i$ can be sure that none of the players $i+1, i+2, \ldots$ will choose the very same site $j$ after player $i$ chooses this site. The player stays out if this highest $p_{j}-c$ in the set of still-empty sites is not positive, or if there is no empty site at this point. (v) In turn, (iv) implies that players fill the most profitable sites (the ones with the largest $p_{j}-c$ ) first, until, sequentially, all sites with $p_{j}-c>0$ are filled, and all players who choose later stay out. The property (iii) also implies that $x_{i j}=0$ for all sites, as no site will be populated by more than one player. Finally, uniqueness for strict order probabilities follows from (iv).

Proposition 5 shows that the efficient equilibrium characterized in Proposition 3 emerges as the sequential equilibrium. Coordination as required for the results of simultaneous site choice seemingly is a strong requirement. But the fact that sequential site choice leads to the same equilibrium allocations may provide a justification. The analysis of sequential site choices also confirms the importance of possible intra-site competition. The possibility of such competition among local competitors at the same sites makes it unattractive for searchers to cluster at the same site, even if this site is likely to carry the prize. The efficiency-enhancing effect of possible intra-site competition does not depend on site choices occurring simultaneously or sequentially. 


\section{Conclusions}

Duplication in delegated research or design contests has always been a major concern. E\&K (2012) took an innovative approach to address this question. For this purpose, they focused exclusively on "where" to search, that is, the choice of design strategy. They find that the non-cooperative coordination equilibrium is characterized by duplication of search effort and too little diversification. Searchers cluster in the most promising search strategies rather than to spread out and efficiently make use of other existing search strategies. Hence, duplication may occur in parallel with some promising search approaches not being considered at all. They advocate multiple prizes as an efficiency improving device. We showed that the efficiency problems of clustering and/or too little diversification can be overcome by a very simple competition mechanism that is likely to be in place in practical cases and could easily be installed if not. If searchers who cluster in the same site react to this and expend effort trying to outcompete their local competitors, this competition can effectively counterbalance the incentives that would otherwise lead to clustering. These results suggest that competition among local competitors, although wasteful if it actually takes place, has an important efficiency enhancing function. It deters would-be competitors from entering into a situation of local competition and induce efficient decision-making about entry and diversification in search strategy choice.

\section{References}

[1] Adamczyk, Sabrina, Angelica C. Bullinger and Kathrin M. Möslein, 2012, Innovation contests: a review and classification outlook, Creativity and Innovation Management, 21(4), 335-360.

[2] Baye, Michael R., Casper G. de Vries and Dan Kovenock, 1996, The all-pay auction with complete information, Economic Theory, 8(2), 291-305.

[3] Boudreau, Kevin J., Nicola Lacetara, and Karim R. Lakhani, 2011, Incentives and problem uncertainty in innovation contests: An empirical analysis, Management Science, $57(5), 843-863$.

[4] Cardon, James H., and Dan Sasaki, 1998, Preemptive search and R\&D clustering, RAND Journal of Economics, 29(2), 324-338. 
[5] Chatterjee, Kalyan, and Robert Evans, 2004, Rivals' search for buried treasure: competition and duplication in R\&D, RAND Journal of Economics, 35(1), 160-183.

[6] Clark, Derek J., and Christian Riis, 1996, A multi-winner nested rent-seeking contest, Public Choice, 87(1-2), 177-184.

[7] Clark, Derek J., and Christian Riis, 1998, Competition over more than one prize, American Economic Review, 88(1), 276-289.

[8] Congleton, Roger D., Arye L. Hillman, and Kai A. Konrad (eds.), 2008, 40 Years of Research on Rent Seeking, volumes 1 and 2, Springer, Heidelberg.

[9] Erat, Sanjiv, and Vish Krishnan, 2012, Managing delegated search over design spaces, Management Science, 58(3), 606-623.

[10] Fershtman, Chaim, and Ariel Rubinstein, 1997, A simple model of equilibrium in search procedures, Journal of Economic Theory, 72(2), 432-441.

[11] Gaba, Anil, Ilia Tsetlin and Robert L. Winkler, 2004, Modifying variability and correlations in winner-take-all contests, Operations Research, 52(3), 384-395.

[12] Garfinkel, Michelle, and Stergios Skaperdas (eds.), 2012, The Oxford Handbook of the Economics of Peace and Conflict. Edited by Michelle R. Garfinkel and Stergios Skaperdas. Oxford University Press, New York.

[13] Glazer, Amihai, and Refael Hassin, 1988, Optimal contests, Economic Inquiry, 26(1), 133-143.

[14] Hutter, Katja, Julia Hautz, Johann Füller, Julia Mueller and Kurt Matzler, 2011, Communication: the tension between competition and collaboration in community-based design contests, Creativity and Innovation Management, 20(1), 3-21.

[15] Hvide, Hans K., 2002, Tournament rewards and risk taking, Journal of Labor Economics, 20(4), 877-898.

[16] Hvide, Hans K. and Eirik G. Kristiansen, 2003, Risk taking in selection contests, Games and Economic Behavior, 42(1), 172-179.

[17] Konrad, Kai A., 2009, Strategy and Dynamics in Contests, Oxford University Press, Oxford. 
[18] Konrad, Kai A. and Dan Kovenock, 2012, The lifeboat problem, European Economic Review, 56(3), 552-559.

[19] Lazear, Edward P., and Sherwin Rosen, 1981, Rank-order tournaments as optimum labor contracts, Journal of Political Economy, 89(5), 841-864.

[20] Lee, Tom, and Louis L. Wilde, 1980, Market-structure and innovation - reformulation, Quarterly Journal of Economics, 94(2), 429-436.

[21] Loch, Christoph H., Christian Terwiesch and Stefan Thomke, 2001, Parallel and sequential testing of design alternatives, Management Science, 47(5), 663-678.

[22] Loury, Glenn C., 1979, Market structure and innovation, Quarterly Journal of Economics, 93(3), 395-410.

[23] Moldovanu, Benny, and Aner Sela, 2001, The optimal allocation of prizes in contests, American Economic Review, 91(3), 542-558.

[24] Moldovanu, Benny, and Aner Sela, 2006, Contest architecture, Journal of Economic Theory, 126(1), 70-96.

[25] Rosen, Sherwin, 1986, Prizes and incentives in elimination tournaments, American Economic Review, 76(4), 701-715.

[26] Simmonds, Norman W., 1985, Duplication of research: a good or a bad thing? Journal of Operational Research Society 36(1), 55-59.

[27] Skaperdas, Stergios, 1992, Cooperation, conflict, and power in the absence of property rights, American Economic Review, 82(4), 720-739.

[28] Szymanski, Stefan, and Tommaso M. Valletti, 2005, Incentive effects of second prizes, European Journal of Political Economy, 21(2), 467-481.

[29] Tullock, Gordon, 1967, The welfare cost of tariffs, monopolies, and theft, Western Economic Journal, 5, 225-232.

[30] Tullock, Gordon, 1980, Efficient rent seeking, in: James Buchanan, Roger Tollison, and Gordon Tullock (eds.), Towards a Theory of the Rent-Seeking Society, Texas A\&M University Press, College Station, Texas, 97-112. 